\title{
Biogenic synthesis of silver nanoparticles using Choerospondias axillaris and its synergistic action with streptomycin
}

\author{
Gunjan Bisht ${ }^{1, ~ *, ~ H i m r a j ~ P a r a j u l i ~}{ }^{2}$, Roshan Baral ${ }^{2}$, Ritesh Thapa ${ }^{3}$ \\ ${ }^{1}$ Department of Natural Science, Kathmandu University, Dhulikhel, Nepal \\ ${ }^{2}$ Deapartment of Biotechnology, SANN International College, Kathmandu, Nepal \\ ${ }^{3}$ Departmant of CPRC, SGCP, Dhapakhel, Nepal
}

Email address:

gunjanbisht31@gmail.com (G. Bisht)

\section{To cite this article:}

Gunjan Bisht, Himraj Parajuli, Roshan Baral, Ritesh Thapa. Biogenic Synthesis of Silver Nanoparticles Using Choerospondias Axillaris and its Synergistic Action with Streptomycin. American Journal of Nanoscience and Nanotechnology. Vol. 2, No. 3, 2014, pp. 50-55. doi: $10.11648 /$ j.nano.20140203.14

\begin{abstract}
This paper mainly focus on combined action of green as well as citrate synthesized silver nanoparticles (AgNPs) and streptomycin, an antibiotic. The synergistic actions of citrate stabilized silver nanoparticles (AgNPs@chem) were compared with that of Nepali hog plum Choerospondias axillaris (Lapsi) synthesized silver nanoparticles (AgNPs@plant), together with action of antibiotic on selected bacterial strains of Escherichia coli and Salmonella typhi. This showed an increase in the action of antibiotic. The size of the synthesized silver nanoparticles was measured by Transmission Electron Microscope (TEM) and X-ray diffraction (XRD).
\end{abstract}

Keywords: Antibacterial Activity, Choerospondias Axillaris, Transmission Electron Microscopy, Silver Nanoparticles, Synergistic Effect

\section{Introduction}

A nanoparticle (or nanopowder or nanocluster or nanocrystal) is a microscopic particle with, at least one dimension less than $100 \mathrm{~nm}$. Nanoparticle investigation is currently an area of passionate scientific research due to a wide variety of potential applications in biomedical,optical and electronic fields. Among the Nobel metals, silver (Ag) is the metal of preference in the field of biological systems, living organisms and medicine. There are diverse methods for nanoparticles formation, among which biological methods are considered as safe and economically sound for the nanomaterial fabrication as an alternative to conventional physical and chemical methods [1-4].

Many antimicrobial agents are limited in clinical applications, because of their poor membrane penetration, complications and side-effects especially at higher doses and relatively higher incidence of bacterial infection due to growing resistance of bacteria to conventional antibiotics and due to in judicial use of many antibiotics. So the use of nanomaterials is being increasingly applied for medical uses and is of great interest as an approach to killing or limiting the activity of numerous micro-organisms. AgNPs exposure, show significant changes in the membranes, which are recognized by the formation of "pits" on their surfaces, and finally, result in the formation of pores and cell death in microbes and is recognized as the best nanoparticles for antibacterial activity. The process of preparation of AgNPs by the reduction of $\mathrm{Ag}^{+}$ions by combinations with bio-molecules found in plant extracts has gain a lot of interest. The capabilities of some microorganisms such as bacteria and fungi to direct the synthesis of metallic nanoparticles have been increasingly employed in the hunt for new materials. Currently, the investigation of this fact has regained importance due to increase in bacterial resistance to antibiotics, caused by their overuse [5-8].

In our study, chemical as well as plant mediated or biogenic synthesis processes were used for the synthesis of silver nanoparticles. Biogenic synthesis was done by using fruit extract of Choreospondias axillaris (Nepali hog plum), an indigenous plant species of Nepal; biogenic synthesis process reduced the steps involved in downstream processing; thus, the process becomes more economical and ecofriendly. Synergistic potential of these silver 
nanoparticles helps in the enhancement of antibacterial activity of the antibiotic. The synergistic action of streptomycin with silver nanoparticles can reduce the need for high dosages of antibiotics and minimize their side effects. The present synergistic study clearly demonstrates that antibiotic, biogenically synthesized silver nanoparticles (AgNPs@ plant) and chemically synthesized silver nanoparticles (AgNPs@chem) using sodium citrate exhibit improved bactericidal activities, and more interestingly, a synergistic activity becomes operational when both of the components act together. The synthesized AgNPs were characterized through UV-Vis spectroscopy, Transmission electron microscopy and X-ray differection technique. The antibacterial activities against Escherichia coli and Salmonella typhi were studied by using gel-diffusion method.

\section{Materials and Methods}

\subsection{Synthesis of Silver Nanoparticles}

For biogenic synthesis of silver nanoparticles (Ag-NPs), Nepali hog plum(Choreospondias axillaris) extract was used as the biological source. The extract of Choreospondias axillaris was used for reduction and capping, which was purchased from local market. While in chemical synthesis sodium citrate was used as capping and reducing agent. Silver nitrate $\left(\mathrm{AgNO}_{3}\right)$ was used as precursor in both the methods, which was purchased from Merck (Mumbai, India).

\subsection{Biogenic Synthesis of Silver Nanoparticles}

Biogenic synthesis of AgNPs was done by using fruit extract of Choreospondias axillaris extract. Fruits were thoroughly washed in deionized water, outer seed covering of the fruit was peeled off and the fleshy part was taken out $10 \mathrm{gm}$ of this flashy part was added in $100 \mathrm{~mL}$ of deionized water, boiled for 30 minutes, and filtered through Whattmann no. 1 filter paper. Equal volume of pale white lapsi extracts was added to an equal quantity of $0.1 \mathrm{M}$ aqueous silver nitrate $\left(\mathrm{AgNO}_{3}\right)$ solution by volume and then was kept in the incubator shaker under $37^{\circ} \mathrm{C}$ for 24 hours. During this period color change was observed i.e. from whitish color to the faint yellow color due to the bioreduction of $\mathrm{Ag}^{+}$ions $\left(\mathrm{Ag}^{+}\right.$to $\left.\mathrm{Ag}^{0}\right)$. The reactants were then characterized at intervals to detect the presence of silver nanoparticles. The percentage of silver nanoparticles in the reaction medium was enhanced by three cycles of centrifugation at $10,000 \mathrm{rpm}$ for $3 \mathrm{~min}$ followed by redispersion in deionized water.

\subsection{Lee and Meisel's Method of Synthesizing Silver Nanoparticles}

Chemical synthesis ofAgNPs was done by using Lee and Meisel's method [9]. Aqueous solution of $125 \mathrm{ml}$ silver nitrate $(1.0 \mathrm{mM})$ was heated till it boils and we added $5 \mathrm{ml}$ $1 \%$ citrate of sodium $(1.0 \mathrm{mM})$ in the mixture as a reducing agent. Citrate of sodium can be also used as stabilizing agent at room temperature. The transparent colorless solution was converted to the characteristic pale yellow, which indicates the formation of AgNPs. It was then cooled to room temperature with constant stirring and was stored in brown bottle at very low temperature which was then used for further characterization and applications.

\subsection{Characterization}

UV-Visible spectra analysis was performed on SSI UV2101 Spectrophotometer machine at SANN International College, Kathmandu to observe Surface Plasmon resonance (SPR) exhibited by AgNPs. To study SPR diluted solution of AgNPs was taken as a sample in cuvette. Spectrum was obtained by scanning the sample from 200-600 nm. X-ray powder diffraction technique was used for phase identification of AgNP crystasl and to obtain information on unit cell dimensions. It is the simplest and most widely used method for estimating the average crystallite size from the full width at half maximum (FWHM) of a diffraction peak using the Scherrer equation [4]. The XRD analysis was performed on D2 Phase machine (Bruker Company) at National Academy for Scienct and Technology(NAST), Khumaltar, Lalitpur, Nepal. For this purpose sample of AgNPs@chem and AgNPs@plant were prepared by centrifugation for about $30 \mathrm{~min}$ at 5000rpm. The pellet thus obtained was cast on a glass slide and was left overnight for drying. It was then examined in XRD machine for analysis. TEM analysis was performed to determine the particle size and distribution of nanoparticles. Colloidal AgNPs solution was used as a sample for TEM analysis. TEM analysis was performed on Jeol 1101 at G.B.Pant University of Agriculture and Technology, India.

\subsection{Synergistic Interaction between Silver Nanoparticles and Antibiotic}

Well diffusion method was used to assay the synergistic effect of streptomycin with plant mediated synthesized and citrate synthesized silver nanoparticles for bactericidal activity against test strains on Muller Hinton agar plates. Briefly, a $5 \mathrm{~mm}$ well made by cork-borer was impregnated with different test samples on E. coli and $S$. typhi cultured agar plates. The Cultured agar plates were then incubated for $24 \mathrm{hrs}$ at $37{ }^{\circ} \mathrm{C}$. A zone of inhibition was observed for different test samples and their diameter was measured [10].

\section{Results and Discussion}

\subsection{Synthesis and Characterization}

In this work, silver nanoparticles were synthesized using lapsi (Choreospondias axillaris) and sodium citrate, respectively. When the reducing agents were mixed with aqueous solution of the silver nitrate, they started to convert the color from colorless to yellowish brown due to reduction of silver ion, which indicated the formation of silver nanoparticles [11-12]. The reduction of silver ion 
was mainly due to the presence of phytochemicals such as flavonoids, phenols, and ascorbic acid, present in Choreospondias axillaris extract. The synthesis of silver nanoparticles by reduction of pure $\mathrm{Ag}+$ ions was monitored by measuring the UV-Vis spectrum. The UV-visible analysis showed that the absorption peaks at 418 and 425 nm for AgNPs@chem and AgNPs@plant were mainly due to the surface plasmon resonance (SPR) of silver nanoparticle, and the broadening of the peak indicated that the particles are polydispersed (Fig.1). The peaks at 418 and $425 \mathrm{~nm}$ are in good agreement with the theoretical simulation of SPR using Mie's theory [13] and different experimental results.

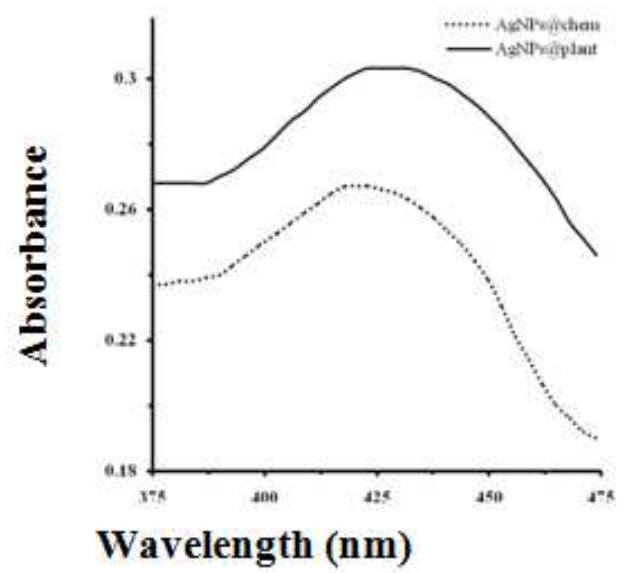

Fig.1.UV-Vis spectral analysis of AgNPs@chemandAgNPs@plant at 1:2 AgNPs/water ratio.

(Fig.2 ) shows the recorded XRD pattern of AgNPs synthesized by both the routes. A number of strong Bragg reflections can be seen which correspond to the (111), (200), (220) reflections of $f c c$ silver in both the AgNPs samples [4]. The XRD results thus show that the silver nanoparticles formed are crystalline. Additionally, the diffractions at around $2 \theta=15^{\circ}$ and $28^{\circ}$ resulting from the biomass or the biomass residue are also notable in the AgNPs@plant sample. The size of the AgNPs was estimated from the Debye-Scherrer formula for both the samples were found to be $28 \mathrm{~nm}$ and $46 \mathrm{~nm}$ by using plant mediated route and chemical route respectively. The size and shape of AgNPs was deduced through transmission electron micrographs at various magnifications ranging 15000-50000X. At 15000X, majority of AgNPs@chem has shown size $50 \mathrm{~nm}$ [Fig. 3(a\&b)]. Subsequent increase in magnification has indicated the prominent presence of Ag@chem with size $50 \mathrm{~nm}$ and irregular shape with some agglomerations. In the same way TEM analysis was performed for Ag@plant at the same magnifications range 15000-50000X. The nanoparticles were evenly dispersed and of different shape [Fig. 3 (c \& d)]. Detailed analysis showed that $\mathrm{Ag} @$ plant appeared as clusters comprising size of $30 \mathrm{~nm}[14]$.

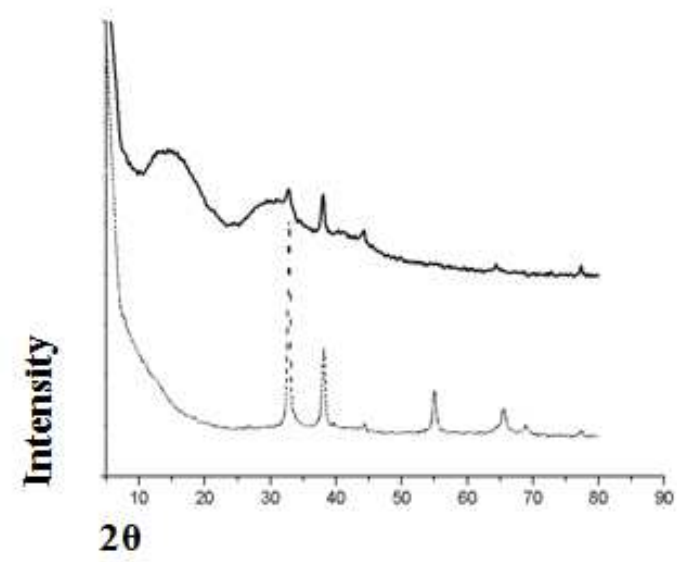

Fig.2. XRD image of AgNPs@chem (- --) and AgNPs@plant ( $\longrightarrow$
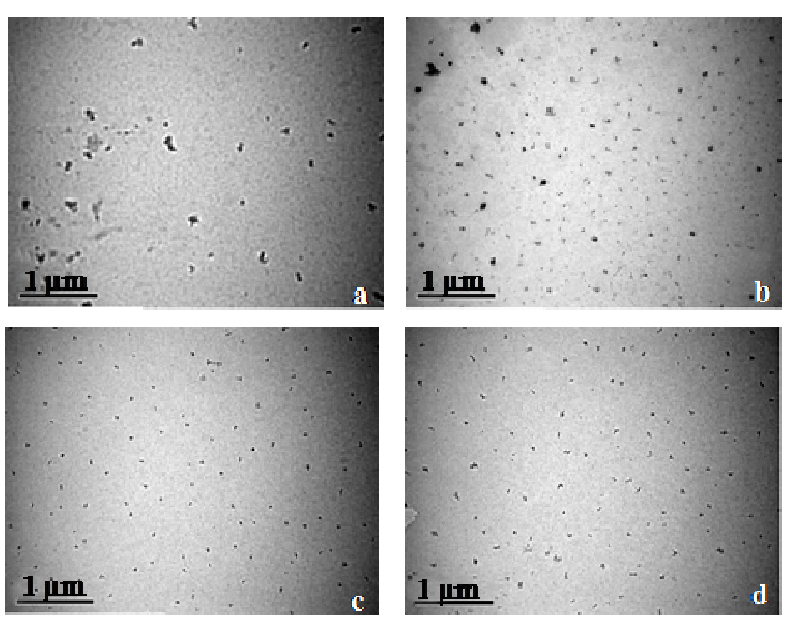

Fig. 3.TEM images of AgNPs@chem (a) and (b) and of AgNPs@plant (c) and (d) at $15000 \mathrm{X}-50000 \mathrm{X}$ magnification.

\subsection{Antibacterial Assay}

The AgNPs synthesized by both routes which was investigated against Gram-negative (Escherichia coli and Salmonella typhi) bacteria in combination with streptomycin antibiotic using the well diffusion method. Both AgNPs@chem as well as AgNPs@plant showed a very slight difference in their antibacterial activities, This implies that an average particle size range from 30-50 nm impose almost similar effect over bacteria or size difference at this range have a very minimal difference in antibacterial activities. The diameters of zone of inhibition (mm) of antibiotic with and without AgNPs against test strains were measured; Streptomycin antibiotic, showed an enhanced antibacterial activity in combination with AgNPs and the $\%$ fold increase in zone of inhibition over streptomycin was recorded; it was found that with AgNPs@chem the antibiotic shows 9.38\% fold increase against Salmonella typhi. Streptomycin also showed a slight enhancement of $6.66 \%$ fold increase against Escherichia coli at (30 $\mu \mathrm{g} / \mathrm{mL})$ of AgNPs@chem (Fig. 4). However,AgNPs@plant (30 $\mu \mathrm{g} / \mathrm{mL})$ showed 16.2\% fold increase against Salmonella typhi, $6.25 \%$ fold increase against Escharichia coli (Fig. 5). 

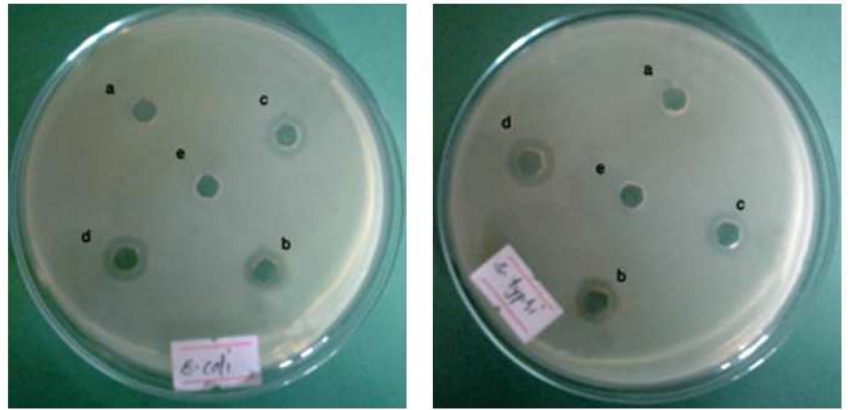

Fig.4. Synergistic activity of streptomycin with AgNPs@chem against Escharichia coli (left) and Salmonella typhi (right); a: AgNO3,b: AgNPs@chem, C: Streptomycin, d:AgNPS@Chem+ Streptomycin, e: Sodium citrate.
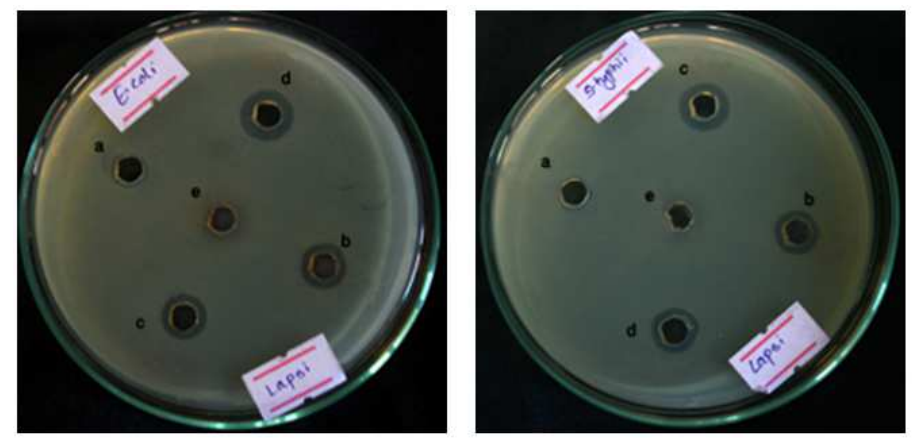

Fig. 5. Synergistic activity of streptomycin with AgNPs@plant against Escharichia coli (left) and Salmonella typhi (right); a: AgNO3, b: AgNPs@plant, C: Streptomycin,d:AgNPS@plant+Streptomycin, e: plant extract.

Table 1and Fig. 4 show the inhibitory effect of different chemicals on the tested strains of bacteria (Escherichia coli and Salmonella typhi). Study against E.coli indicates that the silver nitrate (a) imposes very low antibacterial effect and a very small inhibition zone was formed while the effect was significantly increased with AgNPs@chem $(0.85 \pm 0.13$ mm to $2.8 \pm 0.86 \mathrm{~mm})$. The AgNPs@chem in combination with streptomycin (d) was found to have slight higher inhibitory activity $(3.2 \pm 0.22 \mathrm{~mm})$ than streptomycin (c) alone and
$(3.0 \pm 0.15 \mathrm{~mm})$. Sodium citrate shows no antibacterial effect. Against $S$. typhi enhanced inhibitory activity of AgNPs@chem was found over $\mathrm{AgNO}_{3}$ as the zone of inhibition was increased from $0.67 \pm 0.29 \mathrm{~mm}$ to $3.1 \pm 0.08$ mm. Against $S$. typhi also streptomycin showed enhanced synergistic activity in the presence of AgNPs@chem with $9.38 \%$ fold increase and increases the zone of inhibition from $3.2 \pm 0.16 \mathrm{~mm}$ to $3.5 \pm 0.21 \mathrm{~mm}$. Against both the tested strains sodium citrate showed no effect.

Table 1. Synergistic activity of streptomycin with AgNPs@chem against Escharichia coli and Salmonella typhi.

\begin{tabular}{|c|c|c|c|c|c|c|}
\hline Microrganisms & $\mathrm{AgNO}_{3}$ & AgNPS@Chem & Streptomycin & $\begin{array}{l}\text { AgNPS@Chem } \\
+ \text { Streptomycin }\end{array}$ & Sodium Citrate & \%fold increase * \\
\hline E.coli & $0.85 \pm 0.13$ & $2.8 \pm 0.86$ & $3.0 \pm 0.15$ & $3.2 \pm 0.22$ & Nil & 6.66 \\
\hline S.typhi & $0.67 \pm 0.29$ & $3.1 \pm 0.08$ & $3.2 \pm 0.16$ & $3.5 \pm 0.21$ & Nil & 9.38 \\
\hline
\end{tabular}

*over streptomycin

In the case of AgNPs@plant, streptomycin showed the most pronounced antibiotic synergy against $S$. typhi $16.2 \%$ fold increase, while against E.coli it showed effective synergistic activity of $6.25 \%$ fold increase [Fig. 5]. Study against $E$. coli showed that the silver nitrate (a) impose very low antibacterial effect and gave a inhibition zone of
(1.8 $\pm 0.76 \mathrm{~mm})$ while the effect of AgNPs@plant (b) was nearly double $(3.2 \pm 0.28 \mathrm{~mm})$. The effect of AgNPs@plant (b) and streptomycin (c) alone was found to be almost similar (3.2 \pm 0.28 and $3.2 \pm 0.25)$ respectively shown in Table 2.

Table 2. Synergistic activity of streptomycin with AgNPs@plant against Escharichia coli and Salmonella typhi.

\begin{tabular}{llllll}
\hline Microrganisms & AgNO $_{3}$ & AgNPS@Plant & Streptomycin & $\begin{array}{l}\text { AgNPS@Plant } \\
+ \text { Streptomycin }\end{array}$ & $\begin{array}{l}\text { Plant } \\
\text { Extract }\end{array}$ \\
\hline E.coli & $1.8 \pm 0.76$ & $3.2 \pm 0.28$ & $3.2 \pm 0.25$ & $3.4 \pm 0.37$ & $\begin{array}{l}\text { \%fold } \\
\text { increase }\end{array}$ \\
S. typhi & $1.3 \pm 0.57$ & $3.0 \pm 0.55$ & $3.1 \pm 0.25$ & $3.6 \pm 0.50$ & Nil \\
\hline
\end{tabular}

\footnotetext{
*over streptomycin
} 
Similar pattern of activity was observed for different test chemicals against $S$. typhi. An effective increase in the inhibitory activity of AgNPs@plant was found over $\mathrm{AgNO}_{3}$ same as described earlier the zone of inhibition was increased from $1.3 \pm 0.57 \mathrm{~mm}$ to $3.0 \pm 0.55 \mathrm{~mm}$. Enhanced inhibitory activity of streptomycin was found in the presence of AgNPs@plant with 16.2\% fold increase as the zone of inhibition increases from $3.1 \pm 0.25 \mathrm{~mm}$ to $3.6 \pm 0.50$ $\mathrm{mm}$ when streptomycin was tested alone. Plant extract was not found to show any antibacterial effect against both the tested strains of bacteria.

The antibacterial activity of streptomycin increases in the presence of AgNPs against different test strains, and the synergistic effect of the AgNPs was found to even more prominent than the effect of antibiotics alone. High surface to volume ratio of AgNPs allows them to interact with antibiotics. Moreover, the presence of active groups such as hydroxyl and amido groups in antibiotics may also enable them to interact with nanosilver by chelation which may result in enhanced synergistic effect. More recently, Batarseh's research showed that the bactericidal effect was caused by silver (I) chelating, which prevents DNA from unwinding [15]. The synergistic antibacterial effect with the combination of nanosilver and streptomycin demonstrated more potential, when compared with other antibiotics.

Enhancement of antibacterial activities of antibiotics in combination with AgNPs against different strains of bacterias has already been repoted by many researchers. AgNPs synthesized by green route also showed an increased antimicrobial activity against Staphylococcus aureus, Escherichia coli, Salmonella typhi, and Candida albicans in combination with streptomycin. [16-17]. Recently, Bhande et al. [18] have demonstrated that antibiotics showed enhanced synergism with $\mathrm{ZnO}$ nanoparticles against extended spectrum $\beta$-lactamases producers implicated in urinary tract infections. In their work, they proposed that the combination of $\mathrm{ZnO}$ nanoparticle and antibiotic increased the permeability of membrane which resulted in the leakage of protein from the membrane of bacteria. The results obtained in our study on combined effects of antibiotics with AgNPs are similar to those of earlier studies reported.

\section{Conclusion}

In this study, a new approach of synthesis of AgNPs@plant by using Choerospondias axillaries (Nepali hog plum) extract was developed with conventional chemical method of synthesis. The synthesized nanoparticles were characterized by different analytical techniques viz. UV-Visible spectroscopy to ensure the surface plasmon resonance characteristic colloid AgNPs, XRD analysis for particle size and crystal structure determination and similarly TEM analysis was carried out to study the particle size and its distribution.
The synthesized AgNPs can be applied for a wide range of practical uses, and demonstrates excellent antibacterial performance against Gram-negative E. coli and Salmonella typhi, with much greater efficacy than ionic $\mathrm{Ag}$ or commercial crystalline Ag microparticles. Synergistic study showed that the antibacterial effect of streptomycin was found to be increased in combination with AgNPs synthesized by both the methods. The AgNPs@plant was found to be smaller in size and showed more inhibitory activity than AgNPs@chem in individual study as well as in synergistic study with streptomycin against both the tested bacterial strain. Thus, the developed green synthesis approach by using extract of Choerospondias axillaries provides an effective non-toxic mode of synthesis of AgNPs that can be used as an antibacterial agent.

Finally, in the present work AgNPs synthesized by both the methods exhibited potent antibacterial effects on tested bacteria, probably through destruction of membrane integrity; therefore, it was concluded that AgNPs has considerable antibacterial activity, deserving further investigation for clinical applications.

\section{Acknowledgement}

The authors are highly grateful to the Department of Biotechnology, SANN International College, Gairidhara, Kathmandu, Nepal for providing all the support during the study period.

\section{References}

[1] A. G. Ingale and A. N. Chaudhari, J. Nanomed. Nanotechol., 2013, 4, 2 .

[2] T. P. Amaladhas, S Sivagami, T. A. Devi, N. Ananthiand and S. P.Velammal, Adv. Nat. Sci.: Nanosci. Nanotechnol., 2012, 3, 045006 .

[3] A. M. Fayaz, K. Balaji, M. Girilal, R. Yadav, P. T. Kalaichelvan and R. Venketesan, J. Nanomed.: Nanotechol., Bio. and Med., 2010, 6, 103.

[4] R.Varshney, S. Bhadauria and M. S. Gaur, Adv. Mat. Lett., 2010, 1, 232.

[5] M. Rai, A. Yadav and A. Gade, Biotechnol. Adv., 2009, 27, 76.

[6] H. H. Lara, E. N. Garza-Treviño, L. I. Turrent and D. K. Singh, J. Nanobiotechnol., 2011, 9, 30.

[7] I. Sondi and B. S. Sondi, J. Colloid Interface Sci., 2004, 275, 177.

[8] S. Prabhu and E. K Poulose, Intl. Nano Lett., 2012, 2, 3.

[9] P. C. Lee and D. Meisel, J. Phy. Chem., 1982, 86, 3391.

[10] A. M. Fayaz, K. Balaji, M. Girilal, R. Yadav, P. T. Kalaichelvan, and R. Venketesan, Nanomed., 2010, 6, 103.

[11] K. S. Mukunthan, E. K. Elumalai, T. N. Patel, and V. R. Murty, Asian Pacific J. Tropi. Biomed., 2011, 1, 270. 
[12] N. Hari, T. K. Thomas and A. J. Nair, ISRN Nanotechnol., 2013, 792105.

[13] S. Baset, H. Akbari, H. Zeynali, and M. Shafie, Digest J. Nanomater. and Biostruc., 2011, 6, 709.

[14] W. Li \& X. Xie, Q. Shi , H. Zeng, Y. Yang and Y. Chen, Appl. Microbiol. Biotechnol., 2010, 85, 1115.

[15] K. I. Batarseh, J. Antimicrobiaial Chemotherapy, 2004, 54, 546.
[16] S. P. Dhas, A. Mukherjee, and N. Chandrasekaran, Intl. J. of Pharma. Pharmaceut. Sci., 2013, 5, 292.

[17] M. A. Dar, A. Ingle, and M. Rai, Nanomed., 2013, 9, 105.

[18] R. M. Bhande, C. N. Khobragade, R. S. Mane, and S. Bhande, J. Nanopart. Resear., 2013, 15, 1413. 